\title{
Thematic reviews. Series III: Blood pressure regulation outside the comfort zone
}

\author{
Keith P. George · Paul J. Fadel · Nigel A. S. Taylor
}

Received: 14 October 2013 / Accepted: 9 December 2013 / Published online: 27 December 2013

(C) Springer-Verlag Berlin Heidelberg 2013

Life in general, but physiology in particular, is infinitely more interesting when one moves outside one's comfort zone. This is particularly so when considering the cardiovascular system. Maintaining cardiovascular stability during periods outside the comfort zone (e.g. during exercise) is critical to both performance and health. When one adds multiple stresses together (e.g. exercise in a challenging environment), then our ability to regulate key cardiovascular variables is really put to the test and compromises may often have to be made.

Within cardiovascular and exercise physiology, the regulation and maintenance of arterial blood pressure has received significant research attention, and is often considered as the primary homeostatic variable. The teaching of physiology from an integrated perspective, as opposed to that of the reductionist, requires one to understand not just how blood pressure is regulated to ensure appropriate perfusion, but how the controlled elements of the cardiovascular system are modified when one is faced with stresses that might challenge regulation. In some cases, this may appear in the form of an apparent competition for the available, yet limited, volume of blood. In other cases, individual stresses can, if left unchecked, result in under- or over-perfusion, both with adverse structural, physiological and pathological outcomes.

Communicated by Klaas R. Westerterp/Håkan Westerblad.

\section{K. P. George}

Liverpool John Moores University, Liverpool, London

P. J. Fadel

University of Missouri, Columbia, USA

N. A. S. Taylor $(\varangle)$

University of Wollongong, Wollongong, Australia

e-mail: nigel_taylor@uow.edu.au
In this series of invited reviews, we sought to collect current knowledge and insight in relation to blood pressure regulation, and general cardiovascular function, in response to a number of different, but often overlapping, scenarios. We have invited experts from around the globe to present new data, critical syntheses of past work and insightful reflections on what we know and need to target in future research. There are two particularly important points to note! Firstly, for this series of invited reviews, all of the individual papers are presented in a single volume of the European Journal of Applied Physiology which will act as a fantastic resource to those working or teaching in this field. Secondly, we have an introductory paper from Michael Joyner setting the contextual importance of this topic, and a series summary by Peter Raven and Mark Chapleau that serves as an excellent summary of this broad research area, as well as a call to action for those within this exciting and important field. Enjoy!

Blood pressure regulation: Every adaptation is an integration?

Blood pressure regulation II: What happens when one system must serve two masters-oxygen delivery and pressure regulation?

Blood pressure regulation III: What happens when one system must serve two masters: temperature and pressure regulation?

Blood pressure regulation IV: Adaptive responses to weightlessness.

Blood pressure regulation V: In vivo mechanical properties of precapillary vessels as affected by longterm pressure loading and unloading.

BP regulation VI: Elevated sympathetic outflow with human aging: hypertensive or homeostatic?

Blood pressure regulation VII: The "morning surge" 
in blood pressure: measurement issues and clinical significance.

Blood pressure regulation VIII: Resistance vessel tone and implications for a pro-atherogenic conduit artery endothelial cell phenotype.

Blood pressure regulation IX: Cerebral autoregulation under blood pressure challenges.

Blood pressure regulation $\mathrm{X}$ : What happens when the muscle pump is lost? Post-exercise hypotension and syncope.

Blood pressure regulation XI: Overview and future research directions. 